Original Article

\title{
Effects of Open and Closed Kinetic Chains of Sling Exercise Therapy on the Muscle Activity of the Vastus Medialis Oblique and Vastus Lateralis
}

\author{
Wen-Dien Chang, PhD ${ }^{1}$, Wei-Syuan Huang ${ }^{1)}$, Chia-Lun Lee, PhD²), Hung-Yu Lin, PhD ${ }^{3)}$, \\ PING-Tung LaI, BS $)^{*}$ \\ 1) Department of Sports Medicine, China Medical University, Taiwan \\ 2) Physical Education Section of General Education, National Sun Yat-sen University, Taiwan \\ 3) Department of Occupational Therapy, I-Shou University, Taiwan \\ 4) Department of Physical Therapy and Rehabilitation, Da-Chien General Hospital: No. 6, Shin Guang \\ Street, Miaoli, Taiwan
}

\begin{abstract}
Purpose] The muscle strength of the quadriceps muscle is critical in patellofemoral pain syndrome. The quadriceps muscle supplies the power for dynamic patellar movement, and the vastus medialis oblique (VMO) and vastus lateralis (VL) enable the patella to stabilize during tracking. We followed the theories about open and closed kinetic chain exercises to design two exercises, sling open chain knee extension (SOCKE) exercise and sling closed chain knee extension (SCCKE) exercise. The purpose of our study was to research the changes in quadriceps muscle activity during both exercises. [Methods] Electromyographic analysis was used to explore the different effects of the two exercises. The MVC\% was calculated for the VMO and VL during exercise for analysis. [Results] We found that the mean MVC\% values of the VMO and VL during the SOCKE exercise were higher than those during the SCCKE exercise. The ratio of the VMO to VL was $1.0 \pm 0.19$ during the SOCKE exercise and $1.11 \pm 0.15$ during the SCCKE exercise. [Conclusions] The SOCKE exercise is targeted at quadriceps muscle training and has a recruitment effect on the VMO. The beneficial effect of the SOCKE exercise is better than that of the SCCKE exercise.
\end{abstract}

Key words: Patellofemoral pain syndrome, Sling exercise therapy, Electromyography

(This article was submitted Jan. 23, 2014, and was accepted Feb. 27, 2014)

\section{INTRODUCTION}

Patellofemoral pain syndrome (PFPS) is a common orthopedic disease, and often induces patella pain during climbing and long-distance running ${ }^{1)}$. The results of a previous study indicate that athletes have high risks of knee overuse $^{2)}$. The quadriceps supplies the power of dynamic patellar movement, and the vastus medialis oblique (VMO) and vastus lateralis (VL) enable the patella to stabilize during tracking ${ }^{3)}$. A lack of muscle strength in the VMO decreases the medial muscular tension the patellar and causes the muscle imbalance to increase patellar maltracking ${ }^{4)}$. The ideal ratio of the VMO to VL is $1: 1$, but many PFPS patients have had a low ratio for both muscles in electromyography studies ${ }^{5)}$. Some studies indicated that increasing the muscle strength of the VMO is a useful method of preventing PFPS ${ }^{6,7)}$. Integration of a specific VMO exercise into knee muscle training has been suggested. Some study

*Corresponding author. Ping-Tung Lai (E-mail: steven-mandy@yahoo.com.tw)

(C2014 The Society of Physical Therapy Science. Published by IPEC Inc. This is an open-access article distributed under the terms of the Creative Commons Attribution Non-Commercial No Derivatives (by-ncnd) License $<$ http://creativecommons.org/licenses/by-nc-nd/3.0/>. results indicated that specific VMO exercise training can effectively prevent and reduce the incidence of $\mathrm{PFPS}^{7,8)}$. However, there is still no suitable exercise for VMO muscle activity.

Lower extremity exercise training includes open kinetic chain and closed kinetic chain exercises. The open kinetic chain is a movement in which the distal end of the extremity is not fixed to a relatively stable surface. In the closed kinetic chain, movement of a joint cannot occur without causing predictable movements in the other joints of the extremity ${ }^{9}$. A closed kinetic chain exercise for the knee joint can cause quadriceps and hamstring co-contraction to reduce displacement and improve joint stability, but this will increase the intra-articular pressure ${ }^{10}$. This exercise can cause quadriceps contracture, and this is similar to the muscle activity recruitment occurring during weight-bearing in the gait ${ }^{11)}$. Open kinetic chain exercise uses a lower amount of weight loading for PFPS patients than closed kinetic chain exercise. It is often used for knee rehabilitation in therapeutic exercise. The results of some studies show that closed kinetic chain exercise has a significant therapeutic effect for PFPS patients ${ }^{11-13)}$. However, these studies often focused on the pain decrease and ignored discussing the effect of VMO muscle activity during the exercise training. Sling exercise therapy (SET) is a new training method for athletes 
and orthopedic patients utilized in recent years ${ }^{14)}$. Because of its convenience and practicality, SET has become more and more common in rehabilitation clinics and fitness training centers ${ }^{15}$. However, studies about the effects of using SET with open and closed kinetic chains on VMO muscle training in PFPS patients are rare. We followed the theories about open and closed kinetic chain exercises to design two exercises using SET and explore the VMO and VL muscle activity. To our knowledge, this is the first study to investigate this theme, and we think that the results of this study are important for PFPS patients as well as physical therapists and athletic trainers providing therapeutic exercise.

\section{SUBJECTS AND METHODS}

This study was a cross-over study and was approved by the Institutional Review Board of China Medical University and Hospital. We informed all participants about the experimental process in detail and then obtained their informed consent before the study. We recruited healthy female college students and randomly assigned them to the two SET exercises, comprising sling open chain knee extension (SOCKE) exercise and sling closed chain knee extension (SCCKE) exercise (Fig. 1). A Redcord suspension system (Redcord Trainer, Record, Staubø, Norway) was used to train the quadriceps muscle by one athletic trainer. The two exercises were performed for $10 \mathrm{~min}$, and $3 \mathrm{rep}$ etitions of maximum force were completed in this period. The SOCKE exercise used SET to suspend the knee and maintain knee flexion at 60 degrees. The SCCKE exercise used SET to suspend the ankle and maintain the hip in full extension. We asked the individual to extend the knee to strengthen the concentric contraction of the quadriceps. These exercises were designed to improve the strength of the VMO for application in the field of sports medicine. An electronic goniometer (Flexible 2D goniometer, Noraxon USA Inc., Scottsdale, AZ, USA) was fixed to the lateral knee joint, which was the axis of the goniometer, and the stationary and movable arms were tied to the lateral midline of the thigh and shank. The range of motion of the knee was monitored, and the VMO and VL muscle activity was collected by electromyography by one examiner.

Electromyography (MyoTrace 400, Noraxon USA Inc., Scottsdale, AZ, USA) was used to calculate the maximal voluntary contraction (MVC). The VL electrodes were placed on the line from the anterior superior iliac spine to the superolateral boarder of the patella and $10 \mathrm{~cm}$ from the patella. The VMO electrodes were placed at a distance of $4 \mathrm{~cm}$ from the superior side of the patella along a line inclined $50^{\circ}$ that ran parallel to the line from the anterior superior iliac spine to the superolateral boarder of the patella ${ }^{16)}$. In the electromyography signal processing, the sampling frequency was set to $1,000 \mathrm{~Hz}$. We used a bandpass filter of 40 to $400 \mathrm{~Hz}$ to reduce external noise and performed full-wave rectification. Then, setting the root mean square at $100 \mathrm{~ms}$ smoothed the signal ${ }^{17)}$. Finally, we set the amplitude normalization at $300 \mathrm{~ms}$ and normalized the different movement data by transforming $\mathrm{MVC}$ into $\mathrm{MVC} \%$ with the following formula: $\mathrm{MVC} \%=($ mean $\mathrm{MVC} / \operatorname{maxi}-$

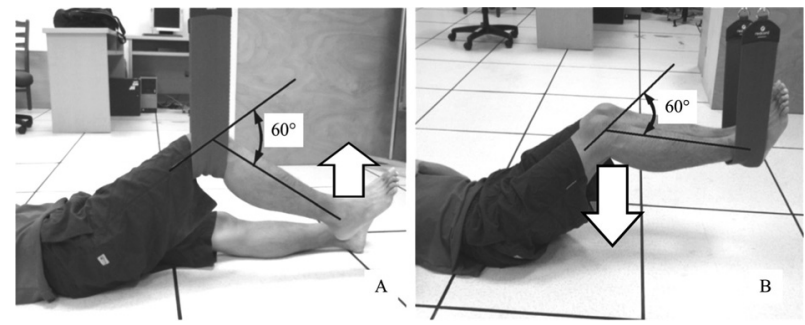

Fig. 1. SOCKE (A) and SCCKE (B) exercises

Table 1. MVC results of the seven participants

\begin{tabular}{ccccc}
\hline & \multicolumn{2}{c}{ SOCKE exercise } & \multicolumn{2}{c}{ SCCKE exercise } \\
\hline no & VMO (\%) & VL (\%) & VMO (\%) & VL (\%) \\
\hline 1 & $98.9 \pm 2.4$ & $84.4 \pm 2.3$ & $79.9 \pm 3.3$ & $63.2 \pm 1.3$ \\
2 & $89.8 \pm 3.1$ & $89.4 \pm 3.1$ & $93.7 \pm 3.2$ & $98.4 \pm 2.8$ \\
3 & $60.6 \pm 3.5$ & $86.3 \pm 4.2$ & $78.6 \pm 2.6$ & $71.2 \pm 2.3$ \\
4 & $99.6 \pm 3.7$ & $98.1 \pm 3.8$ & $82.5 \pm 3.5$ & $81.5 \pm 1.4$ \\
5 & $73.3 \pm 3.2$ & $89.9 \pm 2.7$ & $82.2 \pm 3.3$ & $86.2 \pm 3.3$ \\
6 & $96.5 \pm 2.6$ & $77.1 \pm 3.3$ & $92.1 \pm 4.1$ & $70.1 \pm 2.1$ \\
7 & $85.4 \pm 1.4$ & $78.2 \pm 2.9$ & $90.7 \pm 3.2$ & $74.4 \pm 3.2$ \\
\hline
\end{tabular}

SOCKE, sling open chain knee extension; SCCKE, sling close chain knee extension exercise; VMO, vastus medialis oblique; $\mathrm{VL}$, vastus lateralis

mum force of the resistance test) $\times 100 \%$.

The study data were analyzed using descriptive statistics with SPSS 17.0 (SPSS Inc., Chicago, IL, USA) to compare the activations of the of VMO and VL. The Wilcoxon test was used to compare the differences in the MVO\% values of the VMO and VL between the SCCKE and SOCKE exercises. A 2-tailed test was adopted, and the $p$ value was set at 0.05 . The consistencies of activation of the VMO and VL for 3 repetitions of maximum contraction of one exercise within each individual were analyzed by using the intraclass correlation coefficient (ICC).

\section{RESULTS}

Seven healthy college students (age $=21.3 \pm 0.6$; weight $=51.3 \pm 6.3 \mathrm{~kg}$; height $=161.5 \pm 4.8 \mathrm{~cm}$ ) were recruited and performed 3 repetitions of maximum contraction for each exercise. The ICC for activation of the VMO and VL for 3 repetitions of maximum contraction was $0.91 \pm 0.23$, and there were no significant differences in the SOCKE and SCCKE exercises among the 3 repetitions of maximum contraction. Table 1 shows that 5 participants (Nos. 1, 2, 4,6 , and 7) had higher MVC\% values for the VMO than for the VL during the SOCKE exercise. Five participants (Nos. 1, 3, 4, 6, and 7) had higher MVC values for the VMO than for the VL during the SCCKE exercise. The mean MVC\% values for the VMO $(86.30 \pm 14.61)$ and VL $(86.20$ \pm 7.25 ) during the SOCKE exercise were higher than those for the VMO $(85.67 \pm 6.27)$ and VL $(77.85 \pm 11.81)$ during the SCCKE exercise. No significant differences in $\mathrm{MVC} \%$ values for the VL and VMO were found between the exer- 
cises ( $p>0.05$ ). However, the MVC\% values for the VMO and VL during the SOCKE exercise were higher than during the SCCKE exercise. The $\mathrm{MVC} \%$ of the VL was higher than that of the VMO during both exercises. We also found that the ratio of the VMO to VL was $1.0 \pm 0.19$ during the SOCKE exercise and $1.11 \pm 0.15$ during the SCCKE exercise.

\section{DISCUSSION}

This study compared the VMO and VL muscle activities during the SCCKE and SOCKE exercises. Our results showed that both exercises had a recruitment effect on the VMO muscle. In addition, the SOCKE exercise had higher $\mathrm{MVC} \%$ values for the VMO and VL than the SCCKE exercise. Many studies have used closed kinetic chain and weight-bearing exercises, e.g., double-leg squat and lunge exercise, to improve the VMO strength ${ }^{5,18,19)}$. Their results showed that these exercises can significantly increase the activation of the VMO and VL. Some studies showed that closed kinetic chain exercises can cause greater activation in the VMO than in the $\left.\mathrm{VL}^{5,6}, 19\right)$. However, combining closed kinetic chain exercise with weight-bearing exercise often provides a large increase in pulling strength, and increases the intra-articular pressure. This causes the symptoms of PFPS to worsen during exercise training. We found that the using SET in the SCCKE exercise can also cause greater activation in the VMO than in the VL. SET can reduce the effect of body weight on the knee intra-articular pressure and can be used to train knee extension with nonweight bearing. The SCCKE exercise may be useful and helpful in training VMO activity for PFPS patients. We also found the SOCKE exercise to be more effective in activating the VMO than the SCCKE exercise. Some studies reported that open kinetic chain exercise has little effect on PTPS rehabilitation, because the activation of the VMO was not enough to improve patellar tracking ${ }^{20,21)}$. However, our study results showed that use of SET in the SOCKE exercise can increase the muscle activity of the VMO by more than that of the VL. This beneficial effect is better than that of the SCCKE exercise. We thought suspension of the knee by SET would decrease the postural stability during the SCCKE exercise. This can challenge the stability of the trunk core muscles to facilitate knee extensor contracture. So, our study results showed that the SOCKE exercise, which easily causes quadriceps muscle contraction, can be more effective in training the VMO than the SCCKE exercise.

A previous electromyography study showed that the ideal ratio of the VMO to VL was 1:1 in knee extension exercise for the asymptomatic knee joint ${ }^{5)}$. In our study, the ratios of the VMO to VL in the SOCKE and SCCKE exercises were over the ideal ratio of 1:1. The SOCKE exercise is more suitable training the VMO and VL of PFPS patients than the SCCKE exercise because it has an approaching ideal VMO to VL ratio. All individuals in our study completed the two kinetic chain exercises. The distances between the electromyography pads were also equal. This means that the signals detected for muscle fibers were the same for the
SOCKE and SCCKE exercises. We found that large muscle fibers were recruited for VMO activity during the SOCKE exercise, and the ratio of the VMO to VL was 1.11:1. Previous studies showed high ratios of the VMO to VL at knee extension from 60 to 180 degrees during isokinetic open chain exercise $^{21,22)}$. We used SET to suspend the lower extremity and maintain extension of the knee from 60 to 180 degrees during the SOCKE and SCCKE exercises. Furthermore, our results showed that both exercises can recruit VMO activity, presenting ratios of the VMO to VL higher than 1:1. So, both exercises can improve the VMO muscle contraction of PFSP patients, and the beneficial effect on VMO to VL ratio of the SOCKE exercise is better than that of the SCCKE exercise.

There are still some limitations within our study. First, the healthy individuals in this study did not have pain and symptoms related to the patella and did not conform to the muscle activity of a PTPS patient. Second, the small sample size, 7 participants only, means that the findings cannot be generalized to all PTPS patients. So, we suggest that a larger sample of symptomatic PTPS patients is required in a future study.

\section{ACKNOWLEDGEMENTS}

The authors are grateful for financial support from the National Science Council of the Republic of China and China Medical University under the contract Nos. NSC 102-2815-C-039-091-B and CMU102-SR-21.

\section{REFERENCES}

1) Myer GD, Ford KR, Barber Foss KD, et al.: The incidence and potential pathomechanics of patellofemoral pain in female athletes. Clin Biomech (Bristol, Avon), 2010, 25: 700-707. [Medline] [CrossRef]

2) Tenforde AS, Sayres LC, McCurdy ML, et al.: Overuse injuries in high school runners: lifetime prevalence and prevention strategies. PM R, 2011, 3: 125-131, quiz 131. [Medline] [CrossRef]

3) Powers CM: Patellar kinematics, part I: the influence of vastus muscle activity in subjects with and without patellofemoral pain. Phys Ther, 2000, 80: 956-964. [Medline]

4) Grelsamer RP: Patellar malalignment. J Bone Joint Surg Am, 2000, 82-A: 1639-1650. [Medline]

5) Souza DR, Gross MT: Comparison of vastus medialis obliquus: vastus lateralis muscle integrated electromyographic ratios between healthy subjects and patients with patellofemoral pain. Phys Ther, 1991, 71: 310-316, discussion 317-320. [Medline]

6) Sykes K, Wong YM: Electrical activity of vastus medialis oblique muscle in straight leg raise exercise with different angle of hip rotation. Physiotherapy, 2003, 89: 423-430. [CrossRef]

7) Lam PL, Ng GY: Activation of the quadriceps muscle during semisquatting with different hip and knee positions in patients with anterior knee pain. Am J Phys Med Rehabil, 2001, 80: 804-808. [Medline] [CrossRef]

8) Christou EA: Patellar taping increases vastus medialis oblique activity in the presence of patellofemoral pain. J Electromyogr Kinesiol, 2004, 14 495-504. [Medline] [CrossRef]

9) Floyd RT: Manual of Structural Kinesiology, NY: McGraw-Hill, 2008.

10) Fagan V, Delahunt E: Patellofemoral pain syndrome: a review on the associated neuromuscular deficits and current treatment options. Br J Sports Med, 2008, 42: 789-795. [Medline] [CrossRef]

11) Kaya D, Doral MN, Callaghan M: How can we strengthen the quadriceps femoris in patients with patellofemoral pain syndrome? Muscles Ligaments Tendons J, 2012, 2: 25-32. [Medline]

12) Ismail MM, Gamaleldein MH, Hassa KA: Closed kinetic chain exercises with or without additional hip strengthening exercises in management of patellofemoral pain syndrome: a randomized controlled trial. Eur J Phys Rehabil Med, 2013, 49: 687-698. [Medline] 
13) Bakhtiary AH, Fatemi E: Open versus closed kinetic chain exercises for patellar chondromalacia. Br J Sports Med, 2008, 42: 99-102, discussion 102. [Medline] [CrossRef]

14) Dannelly BD, Otey SC, Croy T, et al.: The effectiveness of traditional and sling exercise strength training in women. J Strength Cond Res, 2011, 25: 464-471. [Medline] [CrossRef]

15) Saeterbakken AH, van den Tillaar R, Seiler S: Effect of core stability training on throwing velocity in female handball players. J Strength Cond Res, 2011, 25: 712-718. [Medline] [CrossRef]

16) Rainoldi A, Melchiorri G, Caruso I: A method for positioning electrodes during surface EMG recordings in lower limb muscles. J Neurosci Methods, 2004, 134: 37-43. [Medline] [CrossRef]

17) Chkeir A, Fleury MJ, Karlsson B, et al.: Patterns of electrical activity synchronization in the pregnant rat uterus. Biomedicine, 2013, 3: 140-144. [CrossRef]

18) Irish SE, Millward AJ, Wride J, et al.: The effect of closed-kinetic chain exercises and open-kinetic chain exercise on the muscle activity of vas- tus medialis oblique and vastus lateralis. J Strength Cond Res, 2010, 24 1256-1262. [Medline] [CrossRef]

19) Coqueiro KR, Bevilaqua-Grossi $D$, Bérzin $F$, et al.: Analysis on the activation of the VMO and VLL muscles during semisquat exercises with and without hip adduction in individuals with patellofemoral pain syndrome. J Electromyogr Kinesiol, 2005, 15: 596-603. [Medline] [CrossRef]

20) Witvrouw E, Danneels L, Van Tiggelen D, et al.: Open versus closed kinetic chain exercises in patellofemoral pain: a 5 -year prospective randomized study. Am J Sports Med, 2004, 32: 1122-1130. [Medline] [CrossRef]

21) Spairani L, Barbero M, Cescon C, et al.: An electromyographic study of the vastii muscles during open and closed kinetic chain submaximal isometric exercises. Int J Sports Phys Ther, 2012, 7: 617-626. [Medline]

22) Tang SF, Chen CK, Hsu R, et al.: Vastus medialis obliquus and vastus lateralis activity in open and closed kinetic chain exercises in patients with patellofemoral pain syndrome: an electromyographic study. Arch Phys Med Rehabil, 2001, 82: 1441-1445. [Medline] [CrossRef] 